Research Article

\title{
Stowage Plan Based Slot Optimal Allocation in Rail-Water Container Terminal
}

\author{
Yanjing Li, Xiaoning Zhu, Li Wang, and Xi Chen \\ School of Traffic and Transportation, Beijing Jiaotong University, Beijing 100044, China \\ Correspondence should be addressed to Xiaoning Zhu; xnzhu@bjtu.edu.cn
}

Received 23 November 2016; Revised 7 December 2016; Accepted 29 December 2016; Published 13 February 2017

Academic Editor: Enrique Onieva

Copyright (C) 2017 Yanjing Li et al. This is an open access article distributed under the Creative Commons Attribution License, which permits unrestricted use, distribution, and reproduction in any medium, provided the original work is properly cited.

To obtain an efficient and reasonable solution for slot allocation in rail-water container terminals, this paper develops storage optimal allocation model 1 to improve the yard space utilization, which is solved by a heuristic algorithm based on Tabu search. Model 2 is then built to reduce the relocation movements. A concept of fall-down problem in shunting operation plan is thus proposed to solve model 2. Models 1 and 2 are tested with numerical experiments. The results show that the yard space utilization increases by $50 \%$ approximately compared to the strategy of one train piling onto a fixed area called a subblock. Meanwhile the number of container relocation movements is less than five when using the fall-down problem strategy. Accordingly, the models and algorithms developed in this paper are effective to improve the yard space utilization and reduce the number of container relocation movements.

\section{Introduction}

The process of container handling in rail-water container terminal can be divided into two parts. In the first part, containers are piled to a certain area in yard space when arriving at the terminal. Generally, there are three types of containers in the storage yard of container terminals, namely, import, export and transshipment containers. Each type of container has its own particular characteristics (as shown in Figure 1), and, what is more, the transshipment containers unloaded from one block container train will be subsequently loaded onto different block container trains, whose operation process is in conformity with export and import containers. Thus, transshipment containers are not considered in this paper.

In the second part, containers piled at the yard space should be retrieved and loaded onto a vessel in accordance with the stowage plan. Containers with highest retrieval priority should be piled at the top of a stack, where containers are piled vertically. As is shown in Figures 2 and 3, container yard in a rail-water container terminal consists of several blocks. The length of each block is comprised of several parallel bays; each bay is composed of a number of stacks. Containers in a certain bay should be unloaded from one train and loaded onto one vessel. But the piling mixed storage solution is meant to pile containers that are to be loaded onto different vessels in a group of bays in different time. Piling separated storage solution means containers from different trains are piled onto different areas. Taking the land scarcity into consideration, piling mixed storage solution is generally preferred with its advantage in improving yard space utilization.

The present research mainly focuses on (1) balancing workloads of containers among blocks when containers are unloaded from a train onto the yard space (as discussed by [1-3]), (2) taking full utilization of single equipment or several equipment (as discussed by $[4,5]$ ) and improving land utilization (as discussed by [6,7]), and (3) reducing the number of overlapping amounts or relocation movements among the bays (as discussed by [8-13]) and solving the storage space allocation problems (as discussed by [4, 14-18]). Pursuit of improving the utilization of yard space leads to an increase in relocation movement. So in order to reduce the number of relocation movements among bays when taking into account the utilization of yard space, it is essential to consider the slot optimal allocation. Here, we set two stages to process the optimization. The first stage is meant to enlarge the number of containers from different trains in each bay during the planning horizon. The second stage is to minimize the number of 


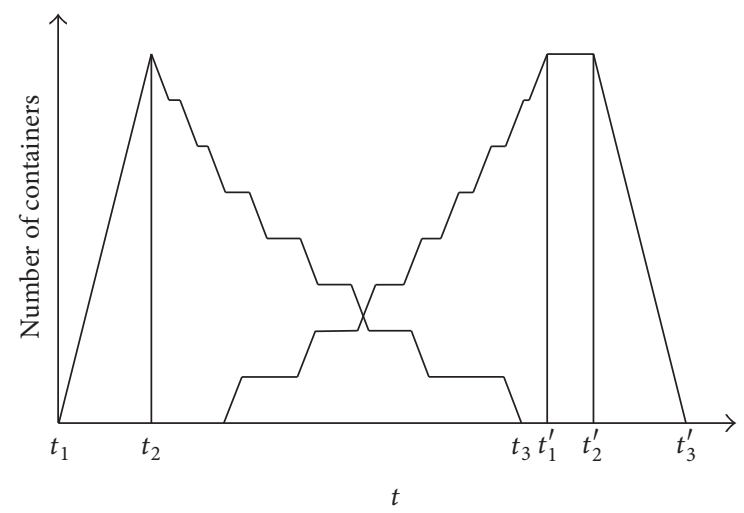

FIGURE 1: Number of containers for vessel at storage yard. $t_{1}$ : starting time of unloading containers from a certain vessel; $t_{2}$ : starting time of loading containers onto several trains; $t_{3}$ : ending time of loading containers onto several trains; $t_{1}^{\prime}$ : ending time of unloading containers from several trains; $t_{2}^{\prime}$ : starting time of loading containers onto a vessel; $t_{3}^{\prime}$ : ending time of loading containers onto a vessel.

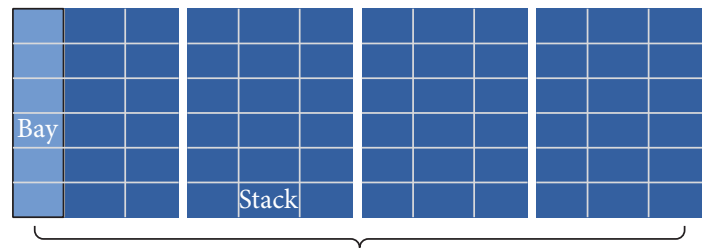

4 blocks

FIGURE 2: The top view of yard space.

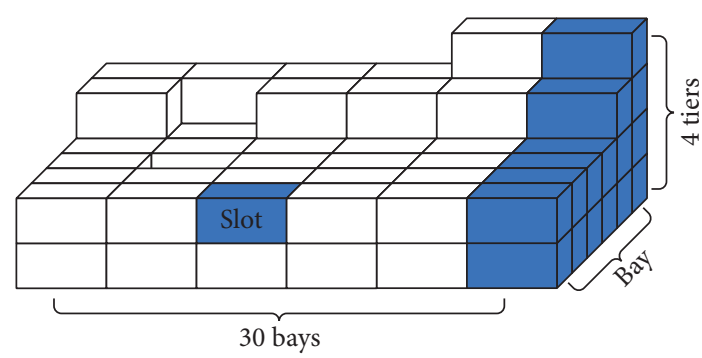

FIgURE 3: The schematic diagram of a block.

relocation movements among bays considering the loading process when the vessel is coming.

The two common indexes for evaluating the container relocation problem can be described as overlapping amount and the relocation movement. The overlapping amount is the number of containers placed above a container retrieved earlier among all the stacks. Relocation movement is the number of containers picked up from the top of a stack and put onto another stack in order to retrieve the highest retrieval priority at present. The retrieval priorities of containers are determined by ship stowage plan.

There are two aspects of this paper when it comes to originality and novelty, namely, the land utilization improvement strategy and the algorithm of reducing relocation movements by referring to the fall-down problem in shunting operation plan.

Firstly, when containers which will be loaded onto one vessel arrive at the terminal, conventional approach is to allocate them onto one fixed area called subblock, and one subblock can only allocate containers belonging to one vessel. Another approach presented in 2012 allocated containers belonging to one vessel onto a subblock consisting of fixed area and shared area. Containers from neighbor subblock can be allocated onto the shared area as long as the shared area is not occupied at the same time. The piling strategy in this paper is to allocate containers belonging to one vessel onto an uncertain area whose size is variable according to the number of containers, and the container occupancy times of the same bays are different; this strategy can be called optimal allocation within the range of space and time.

Secondly, the solving algorithm is proposed by referring to the concept of fall-down problem in shunting operation plan to solve model 2. According to the principle of classification of shunting operations of trains, train detaching and attaching fall-down problem is abstracted as a sequencing problem. When the train detaching and attaching is marshalling after arriving at the terminal, the random-ordered wagon groups formed from the train stock should be turned into ordered wagon groups using the method of humping the random-ordered wagon groups into different tracks; this process can avoid remarshalling when arriving at next station.

A container can be considered as a minimum unit of a train in this algorithm; containers arriving at the terminal earlier and having a higher retrieval priority can be regarded as the random-ordered containers; the random-ordered containers should also be turned into ordered containers using the method of allocating to different stacks; if the number of containers reached the volume of the stack, the containers should be allocated onto another stack which is different from the fall-down problem in shunting operation plan. This approach is only used in shunting operation plan and is never used in slot optimal allocation problem. Here, we firstly use this approach to solve the slot optimal allocation.

\section{Problem Statement}

In this paper, we consider that containers unloaded from several trains onto yard space are to be loaded onto one vessel directly omitting the front storage yard, and the containers are usually stored adjacent to one another to facilitate the loading process. Conversely, since the yard cranes can assign loading operation simultaneously, containers unloaded from a vessel are usually stored separately. At the beginning, a group of bays are empty, and they are gradually filled by incoming containers from a certain train. After loading all the containers onto the vessel, the bays return empty. As shown in Figure 4, the consecutive bays are reserved for a certain vessel, while other containers can be assigned to these bays if only they are occupied in different times.

The current space-sharing concept has two strategies, namely, nonsharing strategy and flexible space-sharing strategy. In the former strategy, each subblock is dedicated to one destination vessel, and the container space can be shared by 


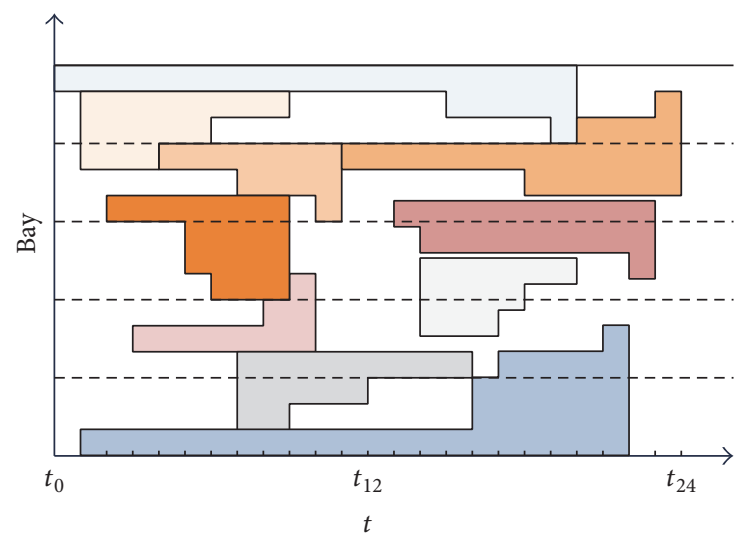

FIGURE 4: The piling strategy of storage yard.

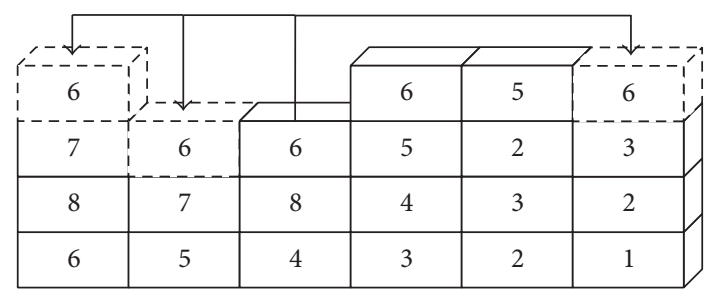

FIGURE 5: Optimization position of relocation movement.

two different vessels as long as their containers do not occupy the space at the same time in the latter one [7]. This paper proposed a completely flexible space-sharing strategy. In this strategy, the number of bays belonging to a certain block varies by the number of containers to be loaded onto a vessel. Each bay can pile any vessels as long as the containers do not occupy the space at the same time.

After choosing which bay to allocate containers, the following process is to pile containers to certain stacks. As Figure 5 shows, one rectangle represents one container, and the number on it represents its retrieval priority. And a larger number indicates a higher retrieval priority. In order to reduce relocation movements, number 8 in third stack should be loaded firstly but number 6 is over it, so number 6 can be moved from the third stack to first or second or sixth stack. When stacking on sixth stack, the second relocation is evitable.

Containers from one vessel are to be unloaded onto several destinations. In each stack, containers retrieval priority varies with the distance of their destination: the farther the destination is, the higher the retrieval priority it has; when it comes to containers with the same destination, heavier containers get the higher retrieval priority according to the stowage plan. Since the destination plays a more important role than the weight in each stack, the level of retrieval is estimated by $G=10 * d+w$, in which $G$ is represented for the retrieval priority, $d$ is in the name of the grade of destination, and $w$ is the level of container weight.

\section{Model Formulations}

The following assumptions are introduced for the development of mathematical models:

(1) The arriving time of each train and the amount of containers are determined and known.

(2) The departure time of every vessel and the amount of containers are determined and known.

(3) Each slot can accommodate one $20 \mathrm{ft}$ container.

Notations which will be used throughout this paper are shown in "Notations."

\subsection{Land Utilization Improvement Strategy}

(1) Objective

$$
\begin{aligned}
& \max \left\{\min \left(S_{k(k+1)}^{t}+S_{(k+1) k}^{t}\right)\right\}, \\
& \forall 1 \leq k \leq K-1,1 \leq t \leq T .
\end{aligned}
$$

The block can be shared by some vessels or trains as long as their containers do not occupy the space at the same time. In this model, it means if the containers going to the same vessel are to be stored in one block with insufficient bays to be piled, the containers can be piled at adjacent bays. The objective is to maximize the sharing yard space at the neighbor blocks.

(2) Constraints

$$
\begin{aligned}
& S_{k(k+1)}^{t} \\
& \leq \begin{cases}C P_{k}-U_{k}^{t}, & k=1, \forall 1 \leq t \leq T, \\
C P_{k}-U_{k}^{t}-S_{k(k-1)}^{t}, & \forall 2 \leq k \leq K-1, \quad \forall 1 \leq t \leq T,\end{cases} \\
& S_{(k+1) k}^{t} \\
& \leq \begin{cases}C P_{k}-U_{k+1}^{t}-S_{(k+1)(k+2)}^{t}, & \forall 1 \leq k \leq K-2, \forall 1 \leq t \leq T, \\
C P_{k}-U_{k+1}^{t}, & k=K-1, \quad \forall 1 \leq t \leq T .\end{cases}
\end{aligned}
$$

Each block has two neighbor blocks except the two blocks at the end of the yard space which only have one neighbor, so the number of bays which can accommodate containers in block $k$ and block $k+1$ in shift $t$ is described in constraints (2).

$$
\begin{gathered}
\sum_{b=1}^{B}\left[\left(r_{k b}^{1}+c_{k b}^{1}\right) \times e_{k b}^{1 t}\right]+\sum_{b=1}^{B} \sum_{t^{\prime}=2}^{t}\left(c_{k b}^{t^{\prime}} \times e_{k b}^{t^{\prime} t}\right)=\sum_{b=1}^{B} E_{k b}^{t}, \\
1 \leq t \leq T, 1 \leq k \leq K, \\
\left(r_{k b}^{1}+c_{k b}^{1}\right) \times e_{k b}^{1 t}+\sum_{t^{\prime}=2}^{t}\left(c_{k b}^{t^{\prime}} \times e_{k b}^{t^{\prime} t}\right)=E_{k b}^{t}, \\
1 \leq t \leq T, 1 \leq k \leq K, 1 \leq b \leq B .
\end{gathered}
$$

The number of containers in each bay (or block) consists of the existing containers piled in each bay (or block) and the 
arriving containers that will be piled in the bay (or block). Constrains (3) calculate the containers in a block at the end of shift under consideration. Constrains (4) calculate the containers in a bay at the end of shift under consideration.

$$
\begin{aligned}
& S_{1,2}^{t}+\sum_{k=2}^{K-1}\left(S_{k(k+1)}^{t}+S_{k(k-1)}^{t}\right)+S_{K(K-1)}^{t} \\
& \quad \leq K \times C P-\sum_{k=1}^{K} \sum_{b=1}^{B} e_{k b}^{t^{\prime} t}, \quad 1 \leq t^{\prime} \leq t-1, \quad 1 \leq t \leq T .
\end{aligned}
$$

A bay can accommodate containers only if the bay is empty. Constraints (5) calculate the maximum number of bays.

$$
\begin{aligned}
& e_{k n}^{t} \times \sum_{n=1}^{N} g_{n}^{t} \leq 1, \\
& 1 \leq k \leq K, 1 \leq b \leq B, 1 \leq t \leq T, \\
& 1 \leq \sum_{k=1}^{K} \sum_{b=1}^{B} e_{k n}^{t} \times g_{n}^{t} \leq x, \\
& {\left[\frac{N\left(g_{n}^{t}\right)}{v_{b}}\right]+1 \leq x \leq X,}
\end{aligned}
$$

$$
1 \leq n \leq N, 1 \leq t \leq T \text {. }
$$

Containers unloaded from a block container train are usually stored in different bays or even different blocks according to the different destination, while a bay can only stack containers from one block container train, as described in constraints (6).

$$
\begin{gathered}
e_{k n}^{t t^{\prime}} \times \sum_{m=1}^{M} h_{m}^{t^{\prime}} \leq 1 \\
\quad 1 \leq k \leq K, 1 \leq b \leq B, 1 \leq t^{\prime} \leq T, \\
1 \leq \sum_{k=1}^{K} \sum_{b=1}^{B} e_{k n}^{t t^{\prime}} \times h_{m}^{t^{\prime}} \leq y, \\
{\left[\frac{N\left(h_{m}^{t^{\prime}}\right)}{v_{b}}\right]+1 \leq y \leq Y,}
\end{gathered}
$$$$
1 \leq m \leq M, 1 \leq t^{\prime} \leq T .
$$

Conversely, the containers to be loaded on a vessel are usually stored together, and a bay can only stack containers to be loaded on one vessel; see constraints (7).

$$
\begin{aligned}
\sum_{b=1}^{B} E_{k b}^{t} \leq V_{k}, \quad 1 \leq k \geq K, \quad 1 \leq t \leq T \\
E_{k b}^{t} \leq V_{b}, \quad 1 \leq k \geq K, \quad 1 \leq t \leq T, \quad 1 \leq b \leq B .
\end{aligned}
$$

Constraints (8) calculate the total amount of containers in a certain block. Constraints (9) calculate the total amount of containers in a certain bay.

\subsection{Relocation Movement Reduction Strategy}

(1) Objective

$$
\min \sum_{z=1}^{Z}\left[W_{z}^{0}+\sum_{l=1}^{L}\left(W_{z}^{l}-W_{z}^{l-1}\right)\right]
$$

In this section, we assume that the problem of retrieving containers is in a single bay. Retrieving containers is managed according to retrieval priority under the consideration of the stowage plan. That is to say, containers from a certain vessel have different destinations: the farther they travel or the heavier they weigh, the higher they should be piled in a stack. The objective is to minimize the further movements.

(2) Constraints

$$
\begin{aligned}
& W_{z}^{l} \\
& =\sum_{x=1}^{J-j^{1}}\left\langle R_{z j^{1}}-R_{z\left(j^{1}+x\right)}\right\rangle+\sum_{x=1}^{j^{n-1}-j^{n}-1}\left\langle R_{z j^{n}}-R_{z\left(j^{n}+x\right)}\right\rangle, \\
& 1 \leq z \leq Z .
\end{aligned}
$$

The containers retrieval follows the rule of "first in last out" in the same stack. And a container at the top of a stack can be retrieved and relocated to other stacks among the bay, only if the container under it has a higher retrieval priority. Constraints (11) commit to calculating the relocation movements considering loading and unloading process. The overlapping amounts may change when container is relocated to other stacks, so it should be recalculated.

$$
W_{z}^{l}-W_{z}^{l-1} \leq W_{\text {else }}^{l}-W_{\text {else }}^{l-1}, \quad 1 \leq z \leq Z, \quad 1 \leq l \leq L .
$$

A container can be moved to another stack in the same bay if it is not fully occupied. Since overlapping amounts will probably increase, which stack to pile is a matter of cardinal significance, as constraints (12) describe.

$$
d_{z j} \geq d_{z(j+1)}, \quad 1 \leq z \leq Z, 1 \leq j \leq J-1
$$

Containers are piled vertically in each stack. If there is no container at the bottom of a stack, all containers cannot be placed above it. It means the container has to be piled from bottom to top in a certain stack. Constraints (13) show the rule of piling containers among stack.

\section{Solution Algorithms}

The reason for proposed algorithm is that our study's problem belongs to the quay crane scheduling problem which was proven to be NP-complete by $[19,20]$; the problem cannot be exactly solved in reasonable time. So we propose a heuristic algorithm to obtain the approximate optimal solution. And Tabu search is dramatically changing our ability to solve a host of problems in applied science and engineering. Tabu search has important links to evolutionary and "genetic" methods, often overlooked. The adaptive memory designs 
of Tabu search have also provided useful alternatives and supplements to the types of memory embodied in neural networks, allowing enhancements of neural network processes in practical settings.

So this section presents a Tabu search algorithm for improving the land utilization problem and a solving algorithm designed by referring to the concept of fall-down problem of shunting operation plan for reducing the relocation movements.

4.1. Tabu Search Algorithm. The procedures of the Tabu search algorithm are as follows.

Step 1. Initial solution $x^{*}$ is described as the optimal solution; namely, $x=x^{*}$; then the objective function value can be calculated as $z^{*}=z\left(x^{*}\right)$; the Tabu list is empty; namely, TaBuList $=\varnothing$; turn to Step 2 .

Step 2. If the termination criterion is met, turn to Step 10; else turn to Step 3.

Step 3. Generate neighborhood spaces of $x$ as $N(x)$ and select a number of solutions as the candidate solution, namely, candidateSolution $(x)$; turn to Step 4.

Step 4. If candidateSolution $(x)=\varnothing$, turn to Step 3; else turn to Step 5.

Step 5. If there is an optimal solution in candidateSolu$\operatorname{tion}(x)$, namely, $z(y) \geq z(x)$, turn to Step 6; else turn to Step 2 .

Step 6. If $y \in T a B u L i s t$, turn to Step 7; else turn to Step 8.

Step 7. candidateSolution $(x)=$ candidateSolution $(x)-\{y\}$, $y=\varnothing$; turn to Step 2 .

Step 8. If $z(y) \geq z^{*}$, then $z^{*}=z(y) ; x^{*}=y$; turn to Step 9; else turn to Step 5 .

Step 9. Update Tabu list; namely, TaBuList $=$ TaBuList $+\{y\}$; turn to Step 10.

Step 10. Output optimal solution $x^{*}$ and the objective function value $z^{*}=z\left(x^{*}\right)$.

The initial solution for Tabu algorithm affects the efficiency of the optimal solution. A group of solutions expressed piling state of containers to be loaded onto each vessel in each shift during planning horizon. The solution is described as $s t=\left(T_{j_{1}}, T_{j_{2}}, \ldots, T_{j_{i}}, \ldots, T_{j_{n}}\right) ; T_{j_{i}}$ is shown as $T_{j_{i}}=$ $\left(T_{b_{1} j_{i}}^{t_{1}}, T_{b_{2} j_{i}}^{t_{2}}, \ldots, T_{b_{x} j_{i}}^{t_{x}}\right)^{\prime}$, which is meant to describe the piling state of containers which are to be loaded onto vessel $j_{i}$. The match value function is shown as $\min \left(\sum_{k=1}^{K} \sum_{b=1}^{B} e_{k b}^{t}\right)$.

The structure of neighborhood solutions determines the form and number of neighborhood solutions. Rules of generating neighborhood solutions are (1) changing the location of two or several trains and (2) removing the locations from one or several trains to another one or several trains. The rules are shown in Figure 6.
Termination criterion of Tabu algorithms contains various methods such as the criterion of frequency control and a certain step of iteration. This paper used the latter one.

4.2. The Algorithm of Reducing Relocation Movements. The second algorithm is to reduce the relocation movements referring to fall-down problem in shunting operation plan; the experimental result of this algorithm is the piling state of each bay; it can be the storage plan when accommodating the containers; it turns out that the relocation movements are decreased. The algorithm of reducing relocation movement has three parts, namely, fall column stage, adjustment stage, and delivering stage. The former two stages are used to accommodate the container to slot; the last stage is to calculate the relocation movements. The procedure of the algorithm is as follows.

\section{(1) Fall Column Stage}

Step 1. Traverse the retrieval priority of containers $i_{l}$ and record the minimum and maximum ones as $N_{\min }$ and $N_{\max }$, search the container where $i_{l}=N_{\min }$, and then initialize the matrix row as $i_{m}=1$; turn to Step 2 .

Step 2. Locate the retrieval priority of container $i_{l}$ as $\left(i_{m}, i_{n}\right)=$ $\left(i_{m}, i_{t}\right)$ in the matrix; record the arriving time and matrix row of last one $L O$ as $T=i_{t}$ and $M=i_{m}$; turn to Step 3 .

Step 3. Update $i_{l}=i_{l}+1$; if $i_{l} \leq N_{\max }$, turn to Step 4; else turn to Step 9.

Step 4. If the retrieval priority of container $i_{l}$ also exists among the earlier arriving containers compared to $L O$, turn to Step 5; else turn to Step 3.

Step 5. If $T<i_{t},\left(i_{m}, i_{n}\right)=\left(M, i_{t}\right)$; else $\left(i_{m}, i_{n}\right)=\left(M+1, i_{t}\right)$; turn to Step 6.

Step 6. If there is container in $M+1$, turn to Step 7; else turn to Step 8.

Step 7. Update the maximum row $M=M+1$ and record the last one's arriving time $T$; turn to Step 3.

Step 8. Update the maximum row $M=M$ and record the last one's arriving time $T$; turn to Step 3.

Step 9. The fall column stage ends.

\section{(2) Adjustment Stage}

Step 1. Initialize $i_{m}=0$; turn to Step 2 .

Step 2. Update $i_{m}=i_{m}+1$; if $i_{m}>i_{\max }$, turn to Step 8; else turn to Step 3.

Step 3. If the number of containers in row $i_{m}$ is larger than volume of each row, namely, $N>j$, delivering the container to another row, turn to Step 4; else turn to Step 2.

Step 4. Initialize $i_{m}^{\prime}=0$; turn to Step 5 . 

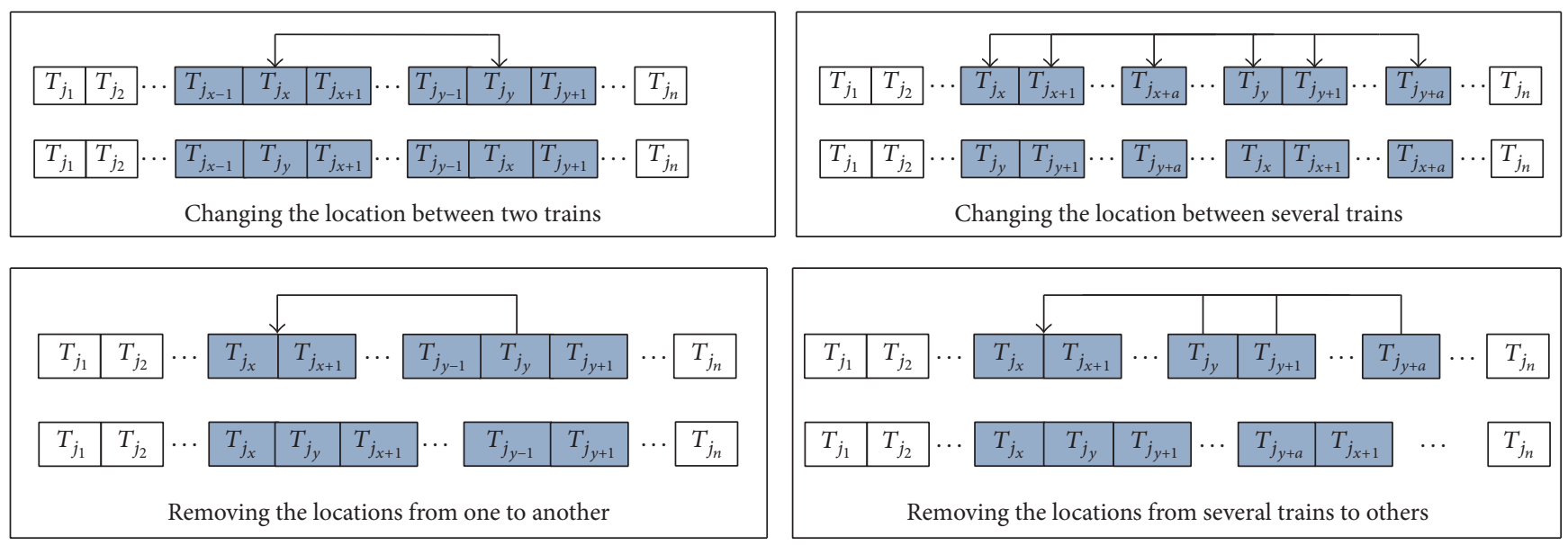

FIGURE 6: The rules of neighborhood solutions.

Step 5. Update $i_{m}^{\prime}=i_{m}^{\prime}+1$; if $i_{m}^{\prime}>i_{\text {max }}$, turn to Step 7; else turn to Step 6.

Step 6. If the number of containers in row $i_{m}$ is smaller than volume of each row, namely, $N<j$, locating the container to row $i_{m}^{\prime}$, namely, $\left(i_{m}, i_{n}\right)=\left(i_{m}^{\prime}, i_{n}\right)$, turn to Step 7; else turn to Step 5 .

Step 7. Compare all the locations that piled the retrieved container; choose a location which has the lowest relocation movements to pile; turn to Step 1.

Step 8. If $i_{m}>i$, turn to Step 9; else turn to Step 14.

Step 9. Select the container of row $N_{\text {min }}$ and then retrieve the container; turn to Step 10.

Step 10. Initialize $i_{m}^{\prime}=0$; turn to Step 11 .

Step 11. Update $i_{m}^{\prime}=i_{m}^{\prime}+1$; if $i_{m}^{\prime}>i_{\max }$, turn to Step 13; else turn to Step 12.

Step 12. If the number of containers for row $i_{m}^{\prime}$ is smaller than volume of each row, namely, $N<j$, locating the container to row $i_{m}^{\prime}$, namely, $\left(i_{m}, i_{n}\right)=\left(i_{m}^{\prime}, i_{n}\right)$, turn to Step 3 ; else turn to Step 11 .

Step 13. Compare all the locations that piled the delivering container; choose a location which has the lowest relocation movements to pile; turn to Step 8.

Step 14. Adjustment stage ends.

\section{(3) Retrieving Stage}

Step 1. Initialize $i_{l}=N_{\max }+1$; relocation movements $r=0$; turn to Step 2.

Step 2. Update $i_{l}=i_{l}-1$; if $i_{l}=N_{\text {min }}$, turn to Step 5; else turn to Step 3.
Step 3. If there is a container with retrieval priority of $i_{l}$, turn to Step 4; else turn to Step 2.

Step 4. Search for the container with retrieval priority of $i_{l}$; calculate the number $(n)$ of containers over it in the same stack; the overlapping amount $r=r+n$; turn to Step 2 .

Step 5. Retrieving stage ends.

\section{Numerical Experiments}

In this section, numerical experiments are carried out to show the performance of land utilization improvement method and the relocation movement reduction method.

There are 24 shifts and the block container train arrives at each shift during the operation horizon. The initial data include the number of containers belonging to each train, the weight of containers, and the destination of containers. The number of containers from each train is a random variable between 50 and 80 . Containers of several trains belong to one vessel, as Table 1 shows. The depth of each block is 6 rows, and the length of it is 30 slots. Piling height is 4 containers high in each stack.

Weight of containers of each train is a variable which approximately obeys normal distribution according to real data. $w \sim N(21.26,3.75)$. As the weight of containers is shown in Table 2, the grade of container weight can be calculated into nine levels; destination of containers from each train is set as a random integer from 1 to 5 which means there are five terminals in one route. The containers' details from the first train are shown in Table 2.

80 bays are needed to accommodate all the containers to be loaded on 7 vessels using the method of piling the containers onto a subblock consisting of a fixed area, as shown in Figure 8; 58 bays are needed using the strategy of piling containers onto a subblock consisting of fixed area and shared area, as shown in Figure 9, while the land utilization improvement strategy in this paper only needs 39 bays, as shown in Figure 7 . The results show that the yard space utilization 
TABLE 1: Volume and arriving time of containers in planning horizon.

\begin{tabular}{lrr}
\hline Arriving time & Number of containers & Vessel \\
\hline$t=1$ & 54 & 6 \\
$t=2$ & 63 & 2 \\
$t=3$ & 68 & 4 \\
$t=4$ & 59 & 7 \\
$t=5$ & 55 & 3 \\
$t=6$ & 71 & 4 \\
$t=7$ & 61 & 4 \\
$t=8$ & 63 & 3 \\
$t=9$ & 52 & 7 \\
$t=10$ & 53 & 7 \\
$t=11$ & 54 & 3 \\
$t=12$ & 51 & 1 \\
$t=13$ & 69 & 3 \\
$t=14$ & 55 & 5 \\
$t=15$ & 71 & 5 \\
$t=16$ & 78 & 6 \\
$t=17$ & 67 & 2 \\
$t=18$ & 58 & 2 \\
$t=19$ & 72 & 1 \\
$t=20$ & 51 & 6 \\
$t=21$ & 53 & 1 \\
$t=22$ & 72 & 2 \\
$t=23$ & 56 & 5 \\
$t=24$ & 56 &
\end{tabular}

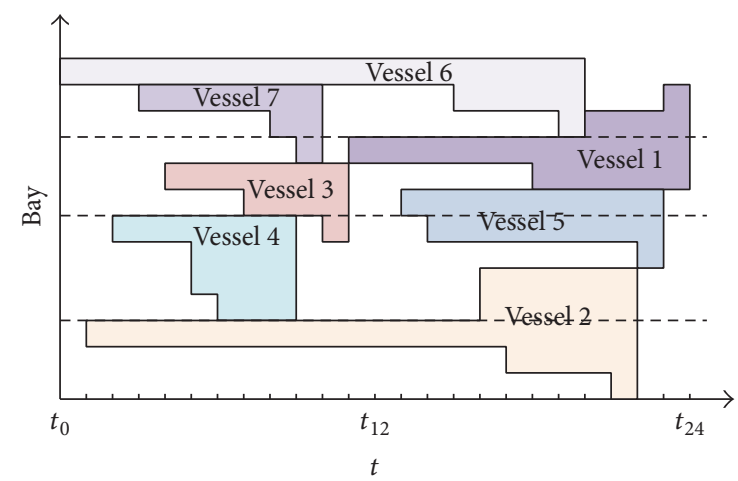

FIGURE 7: The piling state of containers belonging to 7 vessels using the presented method.

increases by $51.25 \%$ compared to the strategy of one train piling onto a fixed area, and the yard space utilization also increases by $32.76 \%$ approximately compared to the strategy of one train piling onto a subblock consisting of fixed area and shared area.

The containers from the first train will be loaded onto the sixth vessel. The piling state in each bay is shown in Figure 10. For example, the container $28_{13}$ at stack 4 of bay 1 is represented where the container will be unloaded at the second destination and the weight of it is $8 ; 13$ represents the arriving
TABLE 2: The containers' details from first train.

\begin{tabular}{|c|c|c|}
\hline Weight & Grade of weight & Destination \\
\hline 17.85 & 3 & 4 \\
\hline 21.12 & 5 & 3 \\
\hline 20.23 & 5 & 3 \\
\hline 19.91 & 5 & 1 \\
\hline 14.3 & 2 & 2 \\
\hline 14.63 & 2 & 3 \\
\hline 20.07 & 5 & 5 \\
\hline 27.39 & 9 & 4 \\
\hline 20.56 & 5 & 4 \\
\hline 20.07 & 5 & 2 \\
\hline 19.98 & 5 & 2 \\
\hline 16.91 & 3 & 4 \\
\hline 26.61 & 8 & 2 \\
\hline 17.99 & 4 & 4 \\
\hline 19.58 & 4 & 3 \\
\hline 14.57 & 2 & 3 \\
\hline 15.54 & 2 & 4 \\
\hline 25.06 & 7 & 1 \\
\hline 24.02 & 7 & 3 \\
\hline 23.45 & 6 & 4 \\
\hline 21.31 & 5 & 5 \\
\hline 17.96 & 4 & 3 \\
\hline 26.95 & 8 & 4 \\
\hline 20.26 & 5 & 3 \\
\hline 21.2 & 5 & 2 \\
\hline 26.37 & 8 & 3 \\
\hline 20.46 & 5 & 2 \\
\hline 20.07 & 5 & 3 \\
\hline 23.87 & 7 & 5 \\
\hline 17.58 & 3 & 4 \\
\hline 21.25 & 5 & 4 \\
\hline 25.23 & 7 & 1 \\
\hline 16.07 & 3 & 1 \\
\hline 20.21 & 5 & 4 \\
\hline 18.34 & 4 & 5 \\
\hline 20.08 & 5 & 3 \\
\hline 16.99 & 3 & 2 \\
\hline 25.56 & 8 & 4 \\
\hline 26.34 & 8 & 3 \\
\hline 16.68 & 3 & 3 \\
\hline 21.13 & 5 & 3 \\
\hline 22.76 & 6 & 2 \\
\hline 21.36 & 5 & 1 \\
\hline 22.78 & 6 & 3 \\
\hline 21.74 & 6 & 4 \\
\hline 20.07 & 5 & 1 \\
\hline 24.36 & 7 & 4 \\
\hline 21.51 & 5 & 2 \\
\hline 21.48 & 5 & 3 \\
\hline 22.41 & 6 & 5 \\
\hline 16.79 & 3 & 4 \\
\hline 12.29 & 1 & 2 \\
\hline 24.24 & 7 & 3 \\
\hline 25.29 & 7 & 4 \\
\hline
\end{tabular}

time. The overlapping amounts and relocation movements for three bays from the first train are shown in Table 3. And the overlapping amounts and relocation movements for each train are shown in Table 4. 


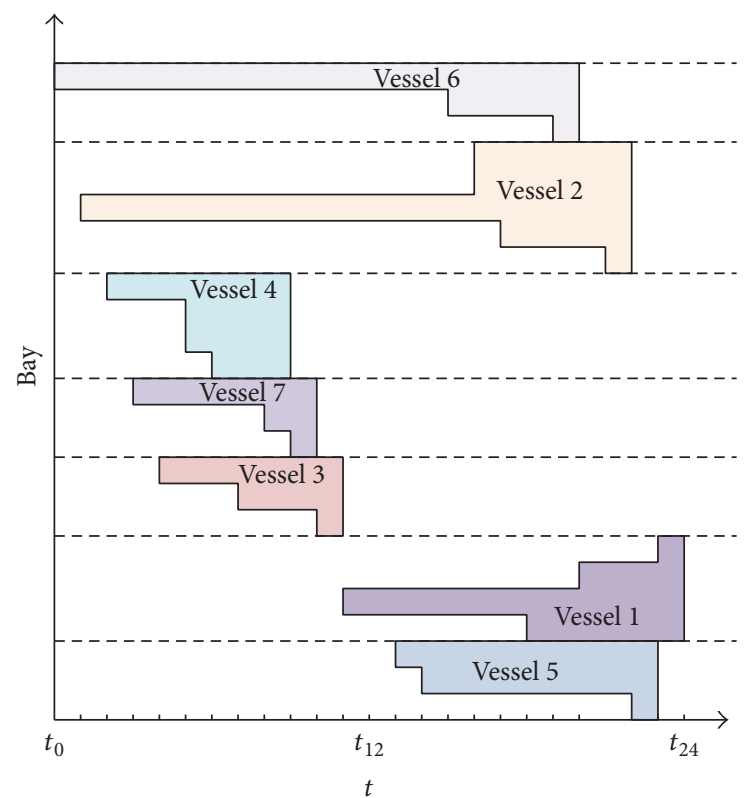

FIGURE 8: The piling state of containers belonging to 7 vessels when piling onto fixed area.

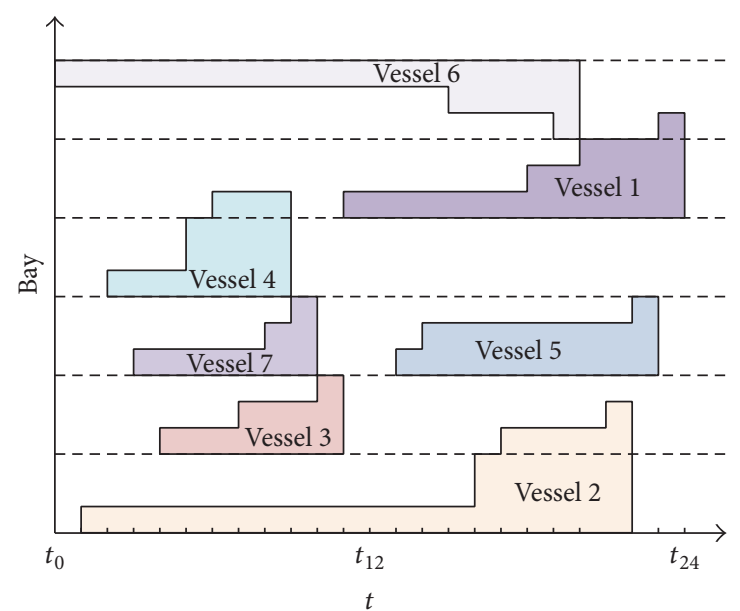

FIGURE 9: The piling state of containers belonging to 7 vessels when piling onto fixed and shared area.

TABLE 3: Overlapping amounts and relocation movements of three stacks from first train.

\begin{tabular}{lcc}
\hline Location & Overlapping amount & Relocation movement \\
\hline Stack 1 & 2 & 3 \\
Stack 2 & 1 & 1 \\
Stack 3 & 1 & 1
\end{tabular}

It was found that slot optimal allocation contributes to reducing relocation movements and making the most use of container yard space. When the number of containers belonging to each vessel increased at every shift, the yard space utilization is improved slightly, and the relocation movements are increased as well. The yard space utilization remains stable but the relocation movements are increased rapidly, when the
TABLE 4: Overlapping amounts and relocation movements for each train.

\begin{tabular}{|c|c|c|}
\hline$t$ & Overlapping amounts & Relocation movements \\
\hline 1 & 4 & 5 \\
\hline 2 & 4 & 4 \\
\hline 3 & 3 & 2 \\
\hline 4 & 3 & 3 \\
\hline 5 & 4 & 3 \\
\hline 6 & 2 & 2 \\
\hline 7 & 1 & 1 \\
\hline 8 & 2 & 3 \\
\hline 9 & 1 & 1 \\
\hline 10 & 4 & 2 \\
\hline 11 & 2 & 2 \\
\hline 12 & 3 & 3 \\
\hline 13 & 2 & 2 \\
\hline 14 & 4 & 4 \\
\hline 15 & 3 & 3 \\
\hline 16 & 4 & 3 \\
\hline 17 & 2 & 2 \\
\hline 18 & 4 & 3 \\
\hline 19 & 5 & 4 \\
\hline 20 & 4 & 3 \\
\hline 21 & 1 & 1 \\
\hline 22 & 2 & 1 \\
\hline 23 & 4 & 3 \\
\hline 24 & 1 & 1 \\
\hline
\end{tabular}

distances to destination (presented as numbers 1 to 5) and the grades of container weight (presented as numbers from 1 to 9 ) rise gradually. When it comes to the delaying time, we examine the variations of the utilization of yard space and the relocation caused by the increase of the delaying time. The results indicate that the delaying time contributes to the former one as it rises, while it makes no sense to the latter one.

\section{Conclusions}

The process for a reasonable container allocation solution consists of two aspects. First, a storage optimal allocation model (model 1) is proposed to share yard space and thus to improve land utilization. Containers from different vessels can be placed at the same area only if their occupancy times of the same bays are different. Second, model 2 minimizes the relocation movements and is solved by a new algorithm referring to the concept of fall column of shunting operation plan. This strategy considers the retrieval priority determined by stowage plan in each stack.

According to the experimental results, the proposed approaches not only improve the yard space utilization but also reduce the relocation movements. That is, they increase the volume of container handling for a fixed amount of storage allocated and decrease the relocation movements that occur during the loading operations of the vessel. In order to simplify the models, we only considered the containers 


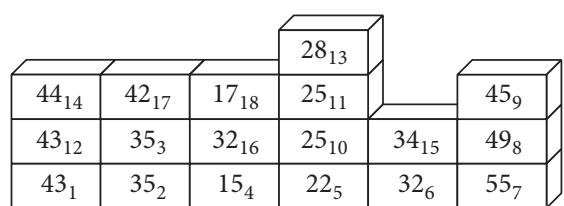

Bay 1

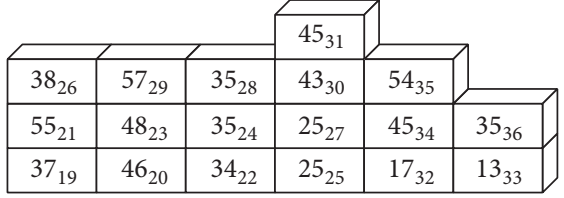

Bay 2

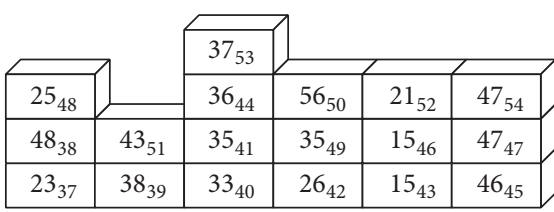

Bay 3

FIgURE 10: The piling state from bay 1 to bay 3 from first train.

from trains which will be loaded onto a vessel in this study. To make it more comprehensive and pragmatic, the opposite direction (from vessel to trains) should be estimated in further discussion.

\section{Notations}

$k: \quad$ Block, which is determined by the maximum number of containers belonging to a single vessel in this model

$S_{k(k+1)}^{t}$ : Number of bays which can accommodate containers in block $k$ and block $k+1$ in shift $t$

$U_{k}^{t}$ : Number of bays which are occupied by containers already stacked in block $k$ in shift $t$

$r_{k b}^{t}: \quad$ Number of containers stacked at the bay $b$ belonging to block $k$ at the end of the shift $t$

$c_{k b}^{t}: \quad$ Number of containers stacked at the bay $b$ belonging to block $k$ arriving in shift $t$

$e_{k b}^{t}: \quad$ It equals 1 , if the container arriving at terminal stack at the bay $b$ belongs to block $k$ at shift $t$

$e_{k b}^{t t^{\prime}}: \quad$ It equals 1 , if the container arriving at terminal stack at the bay $b$ belongs to block $k$ at shift $t$ and still at the same bay at shift $t^{\prime}$

$E_{k b}^{t}: \quad$ Number of containers stacked at bay $b$ belonging to block $k$ in shift $t$

$C P_{k}$ : The set of bays that belong to block $k$; it is a variable

$g_{n}^{t}$ : $\quad$ Block container train $n$ is arriving at the yard space in shift $t$

$N\left(g_{n}^{t}\right)$ : Number of containers from block container train $n$ arriving at the yard in shift $t$

$h_{m}^{t}$ : $\quad$ Vessel $m$ arrives at terminal waiting for loading containers in shift $t$

$N\left(h_{m}^{t}\right)$ : Number of containers to be loaded on vessel $m$ in shift $t$

$V_{k}: \quad$ The maximum number of slots belonging to block $k$

$V_{b}: \quad$ The maximum number of slots belonging to bay $b$

$W_{z}^{l}: \quad$ The overlapping amount in stack $z$ during $l$ times relocation movement; if $l=0, W_{z}^{0}$ represents the amount of overlapping in stack $z$

$W_{\text {else }}^{l}$ : The overlapping amount in each stack except $z$ during $l$ times relocation movement

$\langle a\rangle: \quad$ If $a>0,\langle a\rangle=1$; else $a \leq 0 ;\langle a\rangle=0$

$R_{z j^{n}}: \quad$ The retrieving priority in the tier $j^{n}$ of stack $z$

$d_{z j}$ : when it equals 1 , there is a container in the tier $j$ of stack $z$; when it equals 0 , there is no container in the tier $j$ of stack $z$.

\section{Competing Interests}

The authors declare that they have no competing interests.

\section{Acknowledgments}

This research is subsidized by the Specialized Research Fund for the Doctoral Program of Higher Education of China (no. 20130009110001) and National Natural Science Foundation of China (no. 71390332).

\section{References}

[1] L. Wang, X.-N. Zhu, W. Yan, Z.-Y. Xie, and Q.-B. Li, "Optimization model of mixed storage in railway container terminal yard," Journal of Transportation Systems Engineering \& Information Technology, vol. 13, no. 2, pp. 172-178, 2013.

[2] L. H. Lee, E. P. Chew, K. C. Tan, and Y. Han, "An optimization model for storage yard management in transshipment hubs," $O R$ Spectrum, vol. 28, no. 4, pp. 539-561, 2006.

[3] D.-H. Lee, J. G. Jin, and J. H. Chen, “Terminal and yard allocation problem for a container transshipment hub with multiple terminals," Transportation Research Part E: Logistics \& Transportation Review, vol. 48, no. 2, pp. 516-528, 2012.

[4] X. Yang, N. Zhao, Z. Bian, J. Chai, and C. Mi, "An intelligent storage determining method for inbound containers in container terminals," Journal of Coastal Research, no. 73, pp. 197-204, 2015.

[5] C. Liang, T. Gu, B. Lu, and Y. Ding, "Genetic mechanism-based coupling algorithm for solving coordinated scheduling problems of yard systems in container terminals," Computers \& Industrial Engineering, vol. 89, pp. 34-42, 2015.

[6] X. Jiang, L. H. Lee, E. P. Chew, Y. Han, and K. C. Tan, "A container yard storage strategy for improving land utilization and operation efficiency in a transshipment hub port," European Journal of Operational Research, vol. 221, no. 1, pp. 64-73, 2012.

[7] X. Jiang, E. P. Chew, L. H. Lee, and K. C. Tan, "Short-term space allocation for storage yard management in a transshipment hub port," OR Spectrum, vol. 36, no. 4, pp. 879-901, 2014.

[8] N. Wang, B. Jin, and A. Lim, "Target-guided algorithms for the container pre-marshalling problem," Omega, vol. 53, pp. 67-77, 2015.

[9] C.-J. Liu and Z.-H. Hu, "Multi-objective optimization model for storage location allocation of outbound containers at container yard," Journal of Dalian University of Technology, vol. 55, no. 6, pp. 589-596, 2015.

[10] B. Jin, W. Zhu, and A. Lim, "Solving the container relocation problem by an improved greedy look-ahead heuristic," European Journal of Operational Research, vol. 240, no. 3, pp. 837847, 2015. 
[11] K. H. Kim, Y. M. Park, and K.-R. Ryu, "Deriving decision rules to locate export containers in container yards," European Journal of Operational Research, vol. 124, no. 1, pp. 89-101, 2000.

[12] X. Jiang, E. P. Chew, L. H. Lee, and K. C. Tan, "Flexible spacesharing strategy for storage yard management in a transshipment hub port," OR Spectrum, vol. 35, no. 2, pp. 417-439, 2013.

[13] M. A. Salido, M. Rodriguez-Molins, and F. Barber, "A decision support system for managing combinatorial problems in container terminals," Knowledge-Based Systems, vol. 29, pp. 63-74, 2012.

[14] E. Martin Alcalde, K. H. Kim, and S. S. Marchán, "Optimal space for storage yard considering yard inventory forecasts and terminal performance," Transportation Research Part E: Logistics and Transportation Review, vol. 82, pp. 101-128, 2015.

[15] M. Bazzazi, N. Safaei, and N. Javadian, "A genetic algorithm to solve the storage space allocation problem in a container terminal," Computers and Industrial Engineering, vol. 56, no. 1, pp. 44-52, 2009.

[16] K. H. Kim and K. T. Park, "A note on a dynamic space-allocation method for outbound containers," European Journal of Operational Research, vol. 148, no. 1, pp. 92-101, 2003.

[17] M. Yu and X. Qi, "Storage space allocation models for inbound containers in an automatic container terminal," European Journal of Operational Research, vol. 226, no. 1, pp. 32-45, 2013.

[18] C. Zhang, J. Liu, Y.-W. Wan, K. G. Murty, and R. J. Linn, "Storage space allocation in container terminals," Transportation Research Part B: Methodological, vol. 37, no. 10, pp. 883-903, 2003.

[19] S. H. Chung and K. L. Choy, "A modified genetic algorithm for quay crane scheduling operations," Expert Systems with Applications, vol. 39, no. 4, pp. 4213-4221, 2012.

[20] D.-H. Lee, H. Q. Wang, and L. Miao, "Quay crane scheduling with non-interference constraints in port container terminals," Transportation Research Part E: Logistics and Transportation Review, vol. 44, no. 1, pp. 124-135, 2008. 


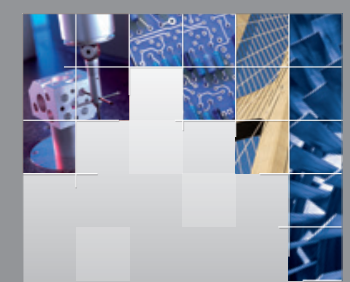

\section{Enfincering}
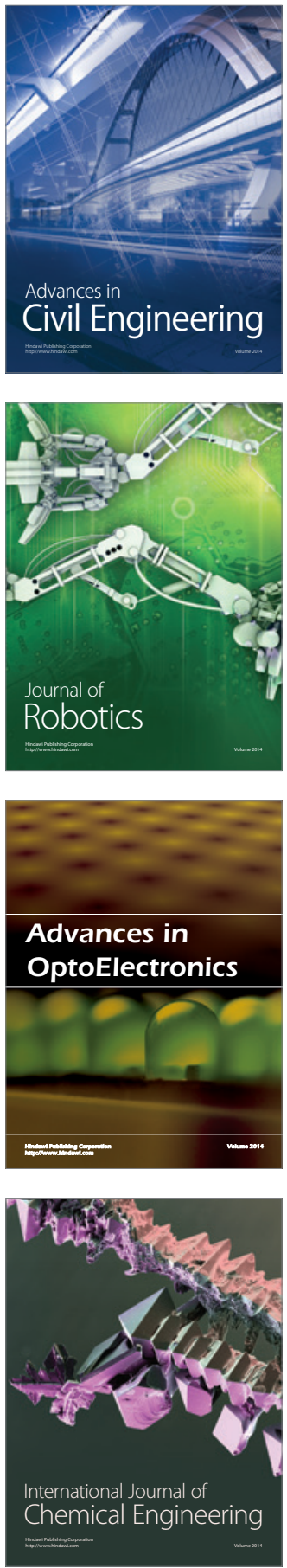

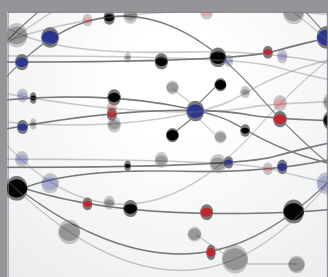

The Scientific World Journal

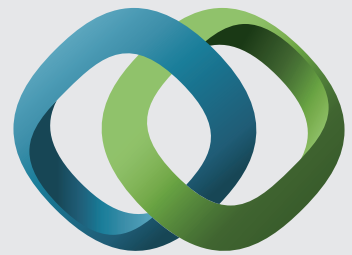

\section{Hindawi}

Submit your manuscripts at

https://www.hindawi.com
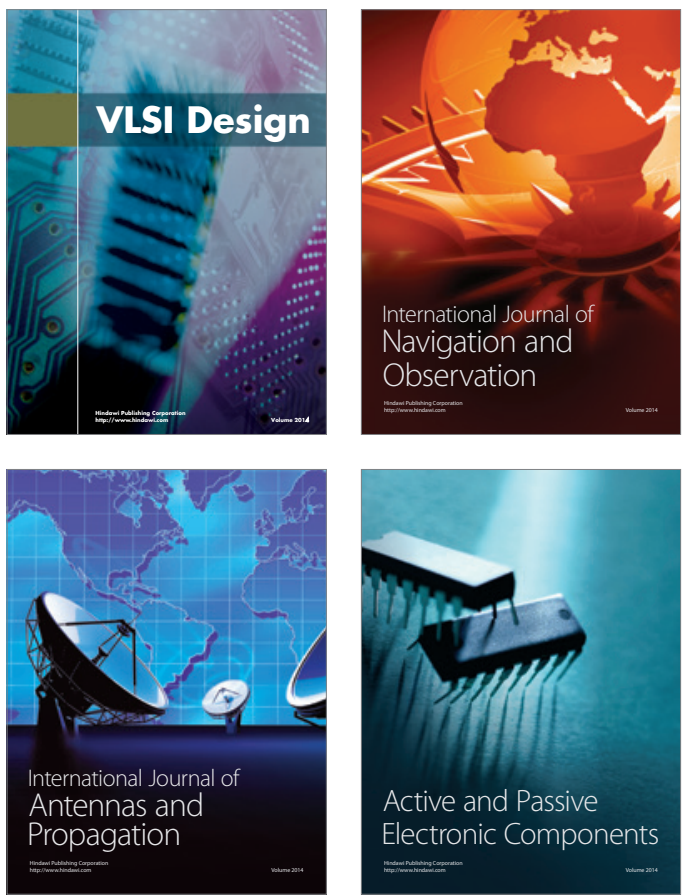
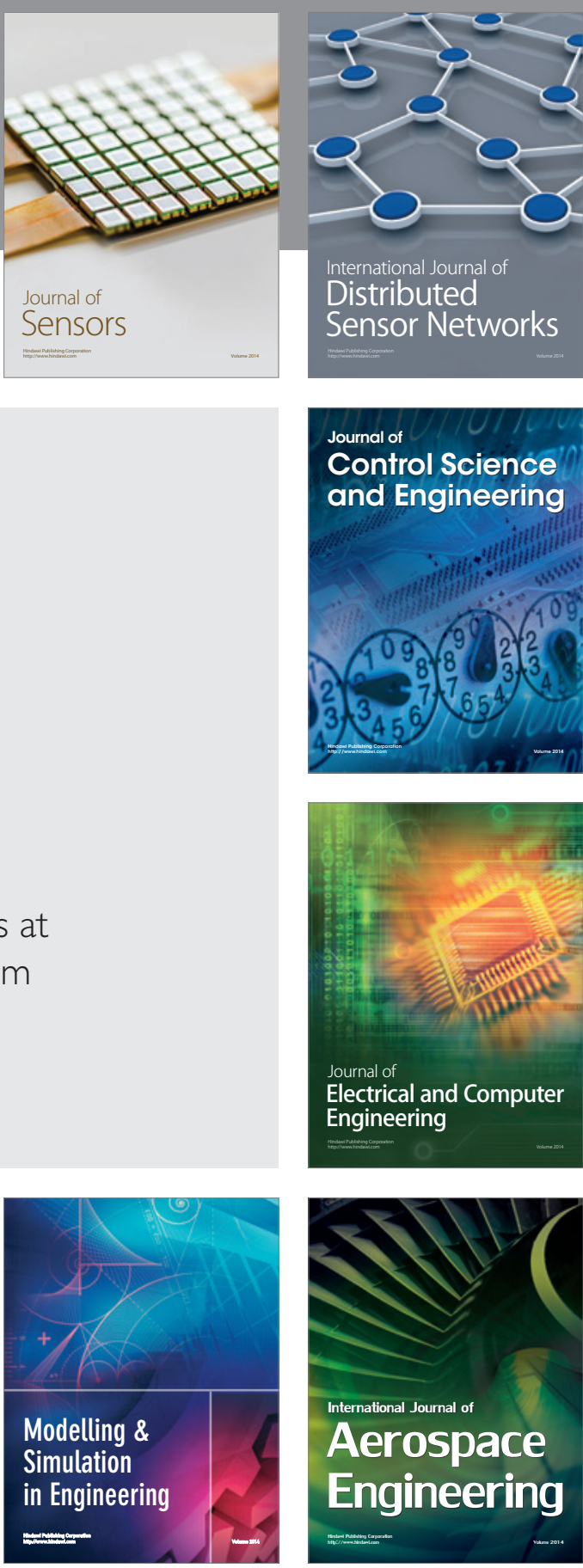

International Journal of

Distributed

Sensor Networks

$-$

Joumal of

Control Science

and Engineering
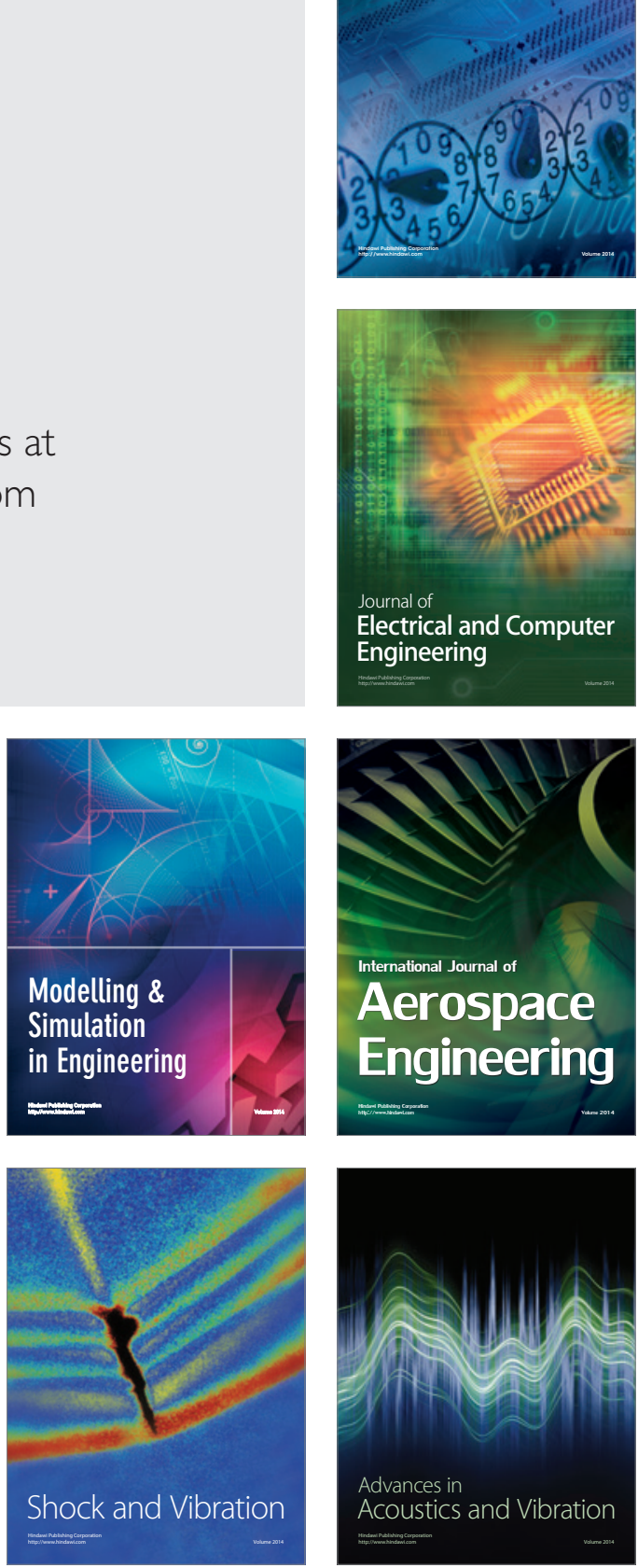